\title{
Estrategias para mejor la calidad educativa con base en el análisis de la trayectoria académica en el área de ingeniería
}

\author{
Strategies to improve educational quality based on the analysis of the \\ academic trajectory in the engineering area
}

\section{Estratégias de melhoria da qualidade educacional a partir da análise da trajetória acadêmica na área de engenharia}

Ivonne Rodríguez Pérez

Universidad Autónoma del Estado de México, México irodriguezperez@yahoo.com https://orcid.org/0000-0003-1819-5881

Rigoberto Pérez Ramírez Universidad Autónoma del Estado de México, México rferezam@yahoo.com.mx https://orcid.org/0000-0003-2982-2676

Flores Albino José Martín Universidad Autónoma del Estado de México, México jmflores@uaemex.mx https://orcid.org/0000-0002-5222-9152

\section{Resumen}

El objetivo de esta investigación fue analizar algunos indicadores de la trayectoria académica de alumnos de ingeniería en Computación (ICO) e ingeniaría en Sistemas y Comunicaciones (ISC) (cohorte 2014-2019) del Centro Universitario UAEM Valle de México. Para ello, se desarrolló un trabajo de tipo no experimental, en el cual se tomaron en cuenta los siguientes indicadores: número de alumnos que concluyeron su plan de estudios, grado de avance, deserción escolar, reprobación, tipo de examen (ordinario, extraordinario y título) mediante el cual los alumnos aprueban cuando están cursando por segunda vez una materia y escuela de donde procedían los estudiantes de ambas ingenierías. Los resultados más destacados 


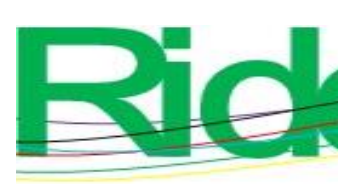

Revista Iberoamericana para la Investigación y el Desarrollo Educativo ISSN $2007-7467$

demuestran que la demanda de ambas ingenierías es equivalente, aunque en cuanto al sexo existe una preponderancia por parte de los hombres. Asimismo, se puede indicar que el grado de avance disminuye en el segundo y tercer semestre de ambas carreras, ya que los alumnos consideran que son los de mayor grado de dificultad. Para atender estas situaciones se recomiendan estrategias de atención que incluyan asesorías, tutorías, canalización psicológica, entre otras.

Palabras clave: deserción escolar, eficiencia, rendimiento escolar, repetición de curso.

\section{Abstract}

The objective of this research was to analyze some indicators of the academic trajectory of students of Computer Engineering (ICO) and Engineering in Systems and Communications (ISC) (2014-2019 cohort) of the UAEM Valle de México University Center. For this, a non-experimental type of work was developed, in which the following indicators were taken into account: number of students who completed their study plan, degree of progress, school dropout, failure, type of exam (ordinary, extraordinary and title) by which students pass when they are taking a subject for the second time and the school where the students of both engineering degrees came from. The most outstanding results show that the demand of both engineering is equivalent, although in terms of sex there is a preponderance on the part of men. Likewise, it can be indicated that the degree of progress decreases in the second and third semesters of both careers, since students consider that they are the ones with the highest degree of difficulty. To attend to these situations, attention strategies are recommended that include counseling, tutoring, psychological channeling, among others.

Keywords: school dropout, efficiency, school performance, grade repetition.

\section{Resumo}

O objetivo desta pesquisa foi analisar alguns indicadores da trajetória acadêmica de alunos de Engenharia de Computação (ICO) e Engenharia de Sistemas e Comunicações (ISC) (coorte 2014-2019) do Centro Universitário UAEM Valle de México. Para tal, foi desenvolvido um tipo de trabalho não experimental, no qual foram considerados os seguintes indicadores: número de alunos que concluíram o plano de estudos, grau de aproveitamento, evasão escolar, reprovação, tipo de exame (ordinário, extraordinário e título ) pela qual os 


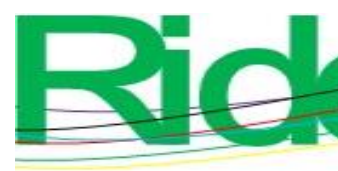

Revista Iberoamericana para la
Investigación y el Desarrollo Educativo
ISSN $2007-7467$

alunos passam quando estão cursando uma matéria pela segunda vez e a escola de onde vieram os alunos dos dois cursos de engenharia. Os resultados mais marcantes mostram que a demanda de ambas as engenharias é equivalente, embora em termos de sexo haja uma preponderância por parte dos homens. Da mesma forma, pode-se indicar que o grau de progressão diminui no segundo e terceiro semestres de ambas as carreiras, uma vez que os alunos se consideram com maior grau de dificuldade. Para atender a essas situações, são recomendadas estratégias de atenção que incluem aconselhamento, tutoria, canalização psicológica, entre outras.

Palavras-chave: evasão escolar, eficiência, desempenho escolar, repetência.

Fecha Recepción: Septiembre 2020

Fecha Aceptación: Febrero 2021

\section{Introducción}

La globalización, el uso de las TIC y el conocimiento han impuesto a la educación superior nuevos retos para transformar la sociedad e impulsar el desarrollo. Esos factores, de hecho, han llevado a reconsiderar si los servicios que brindan a la comunidad son adecuados, por lo que se hace necesario analizar la calidad de la formación que reciben los estudiantes en su paso por la universidad.

La calidad de las instituciones de educación superior (IES) depende de la pertinencia de los programas ofertados, lo cual se puede conocer mediante diversos indicadores de la trayectoria académica, como el rezago, la reprobación, la eficiencia terminal y la deserción. Estos permiten diagnosticar y determinar el impacto de la educación recibida por los alumnos, así como de la eficiencia de las instituciones (González, 1999), lo que en definitiva sirve para elaborar y aplicar alternativas que permitan fortalecer el funcionamiento de la universidad.

En materia de deserción escolar, esta constituye, por su magnitud, un problema importante del sistema nacional de educación. Las altas tasas de abandono de los estudios que se producen en todos los niveles educativos tienen incidencia negativa sobre los procesos políticos, económicos, sociales y culturales del desarrollo nacional.

En América Latina, la desigualdad social es alta y la tasa de deserción oscila entre $40 \%$ y $70 \%$, lo que contribuye a ampliar esta situación. En tal sentido, con frecuencia los estudiantes provenientes de los grupos más vulnerables son los que no terminan sus estudios (Salmi, 2016). 


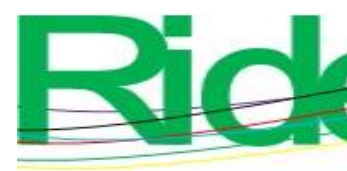

Revista Iberoamericana para la Investigación y el Desarrollo Educativo ISSN $2007-7467$

En el caso de México, algunas de las dificultades que enfrenta la educación superior tienen que ver con la cobertura, la infraestructura, la planta académica, el programa curricular, la calidad de la formación profesional y del profesorado, la producción científica, la eficiencia terminal, entre otros (Rugarcía, 1994). De hecho, algunos estudios han hecho evidente la necesidad de saber más acerca de los estudiantes, sus expectativas, sus intereses y motivaciones, así como de sus problemas y necesidades (Chaín-Revuelta, 1995; Reyes Pérez, 2006; Sánchez, 2006).

En tal sentido, dentro del Plan Sectorial de Educación 2013-2018 del Gobierno federal (en el capítulo 1, relacionado con el Diagnóstico de la educación superior y formación para el trabajo) se señala que el abandono de los estudios en educación superior es uno de los desafíos del sistema educativo. Uno de los criterios para evaluar la calidad de una IES es la eficiencia terminal por cohorte generacional, indicador que se puede medir por los índices de reprobación, deserción y rezago.

Debido a lo anterior, en los años setenta del siglo pasado, dentro la investigación educativa de México surgieron estudios enfocados en la trayectoria académica, las relaciones pedagógicas, la interacción y las experiencias cotidianas en el proceso educativo (De Allende y Gómez, 1989), lo que dio origen a una nueva corriente educativa. De ellos, los estudios de trayectoria escolar se enfocaron en revelar las diferentes problemáticas a las que se enfrenta el estudiante a lo largo de su vida académica. Su análisis proporciona información para diseñar acciones encaminadas a lograr un mejor trayecto del alumno en la institución educativa.

Los primeros trabajos sobre trayectorias académicas en México se concretaron en la década de los ochenta con diseños de carácter descriptivo, los cuales atrajeron la atención de los diseñadores de políticas educativas y de los directivos de las IES. Esto dio paso a que se empezaran a posicionarse en la agenda de investigación temas como el abandono de los estudios, la deserción y la eficiencia terminal.

En las primeras indagaciones se empezaron a identificar las dimensiones del objeto de estudio. De este modo surgió una de tipo institucional, relacionada con la eficiencia interna del sistema, la eficiencia terminal, el rendimiento y los procesos de evaluación institucional ligados al desempeño de los estudiantes. Al mismo tiempo, otra dimensión se enfocó en el comportamiento individual y grupal reflejado en el fracaso escolar, éxito, deserción, rezago, abandono, repetición, entre otros. Lo anterior implicaba conocer cuestiones básicas que 


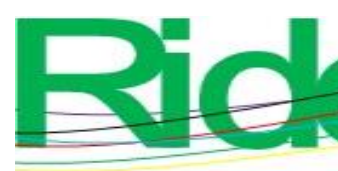

Revista Iberoamericana para la Investigación y el Desarrollo Educativo ISSN $2007-7467$

ayudaran a explicar las actitudes estudiantiles en relación con su ubicación y origen; por ejemplo, identificar de dónde venían, sus hábitos de estudio más comunes, etc.

Ahora bien, debido al contexto donde surgieron algunos de estos estudios (Bartolucci, 1994, 1998; Calatayud y Merino, 1984; Camarena, Chávez y Gómez, 1985; Covo, 1988; Gómez, 1990; Noriega, 1989; Tinto, 1993), la definición de los indicadores se estableció según directrices académicas, administrativas y normativas de las propias instituciones.

Desde el punto de vista cuantitativo, realizar el seguimiento de la trayectoria académica de los estudiantes que ingresan a la universidad permite conocer, entre otras cosas, el comportamiento de la matrícula a través de los semestres e identificar posibles estrategias de acción en función del apoyo académico que pueda proporcionar la institución, como se muestra en seguida.

Por ejemplo, en la Universidad Autónoma de Tamaulipas (UAT) se implementó un modelo analítico big data, el cual considera que la deserción escolar es un problema multifactorial que no se puede atender con el análisis de información limitada. Este modelo permite cruzar e integrar datos de diferentes asociaciones, organizaciones e instituciones externas con datos de los propios sistemas de información institucionales con el objetivo de identificar las causas y factores que inciden en la deserción escolar universitaria (UAT, 2016).

En el caso de la licenciatura en Psicología de la Universidad Nacional Autónoma de México (UNAM), se considera que los problemas de rezago y deserción son complejos y tienen causas multifactoriales, por lo que las alternativas para disminuir estos índices y mejorar la calidad de la educación requieren la participación conjunta y coordinada de los sectores implicados en el proceso educativo: alumnos, administrativos y profesores. En esta IES se estima como esencial identificar las ventajas y limitaciones del Programa Institucional de Tutorías, el cual necesita contar con un mecanismo para seguir y evaluar los efectos y el impacto del programa en el rendimiento académico. Otra propuesta en la referida casa de estudios es seguir con las actividades del Centro de Atención y Seguimiento al Desarrollo Académico (CASDA) para apoyar y orientar a los alumnos en cuanto al desarrollo de estrategias de estudio, comprensión lectora y otros temas asociados al desempeño académico (UNAM, 2015).

De esta manera, al detectar a los alumnos con niveles de riesgo de abandonar los estudios, los esfuerzos podrán priorizar la atención, de manera correctiva, ayudando a quienes se encuentran con niveles altos de riesgo escolar; como de manera preventiva apoyando a los alumnos sin riesgo a permanecer en este nivel. 


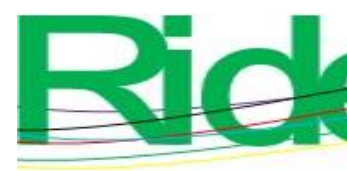

Revista Iberoamericana para la
Investigación y el Desarrollo Educativo
ISSN $2007-7467$

En concordancia con las ideas anteriores, la Universidad Autónoma del Estado de México (UAEM) plantea en el Plan Rector de Desarrollo Institucional (PRDI) lo siguiente: La Universidad Autónoma del Estado de México, al igual que otras instituciones de educación superior, tiene establecidos estándares de calidad y los medios para conseguirlos. Se espera que esos mecanismos permitan, entre otros logros, disminuir el abandono escolar y la reprobación, para incrementar la eficiencia terminal, lo cual redundará en la formación de profesionales competentes, creativos e innovadores (UAEM, 2017a, p. 65).

Al hablar de calidad en la formación de nuestros estudiantes es indispensable analizar los indicadores que dan cuenta de ello. Uno de estos indicadores es el índice de reprobación en estudios profesionales; este permite deducir la eficiencia del proceso educativo (aprovechamiento) e induce a buscar referencias contextuales (sociales y económicas, básicamente) de los alumnos que ingresan en este esquema de reprobación y de fallas posibles en el proceso de enseñanza-aprendizaje (UAEM, 2017a, p. 66).

Con base en los preceptos anteriores, la UAEM en 2004 implementó el Modelo Institucional de Innovación Curricular (MIIC), el cual tiene como fundamento la flexibilidad y la enseñanza basada en competencias y en el estudiante. El MIIC sienta las bases para que la formación de los profesionales responda y se ajuste permanentemente a las demandas sociales y a los avances científicos, humanísticos y tecnológicos, y promueva la articulación equilibrada del saber (conocimientos), saber hacer (procedimientos), saber ser (valores), que propicien un pensamiento crítico y desarrollen la capacidad de solucionar problemas tanto en el contexto teórico disciplinar como en el social (campo real, inserción de la profesión), con una visión interdisciplinaria y transdisciplinaria (Moreno, Medina, Espinoza y Miranda, 2005).

Asimismo, se inserta al Plan General de Desarrollo de la Universidad, pues estrechamente vinculado con la deserción se encuentra el problema de la reprobación, es decir, el porcentaje de estudiantes que no logran adquirir el mínimo de conocimientos necesarios para acreditar la asignatura cursada dentro de los planes fijados para ello.

Actualmente, según cifras oficiales, más de $30 \%$ de los estudiantes de media superior presentan dificultades para adquirir satisfactoriamente los conocimientos y habilidades en distintas asignaturas, en especial en disciplinas como matemáticas, física, química, inglés y biología. Por eso, el Programa Sectorial de Educación ha trazado, para 2021, la meta de 


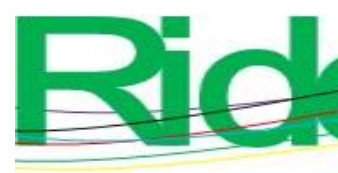

Revista Iberoamericana para la Investigación y el Desarrollo Educativo ISSN $2007-7467$

disminuir el índice de reprobación de $30 \%$ a $18 \%$ en este nivel educativo, con especial énfasis en las áreas señaladas (UAEM, 2009).

Finalmente, se puede señalar lo indicado dentro del Plan de Desarrollo del Centro Universitario Valle de México (PDCUVM), donde "para apoyar a los alumnos que presentan rezago, se implementaron 18 cursos remediales y mentorías académicas, en caso de reprobación, grupos especiales y cursos intensivos”(UAEM, 2017b, p. 21).

\section{Trayectoria académica}

La trayectoria académica se refiere a la cuantificación del comportamiento escolar de un conjunto de estudiantes con características similares (cohorte) durante su trayecto o estancia educativa (es decir, desde el ingreso hasta la conclusión de los créditos y los requisitos académico-administrativos que definen el plan de estudios) (Ponce de León, 2003). Este constructo también incluye el conjunto de factores y datos que afectan y dan cuenta del comportamiento escolar de los estudiantes durante su estancia en la universidad (Fernández, Peña y Vera, 2006).

Chaín-Revuelta (1995) explica que las trayectorias escolares exigen diferenciar un conjunto de cuestiones que van desde la eficiencia interna, la eficiencia terminal y el rendimiento hasta los comportamientos académicos de los estudiantes durante su vida escolar (como el aprovechamiento académico, el fracaso, el éxito, el logro, la aprobación, la reprobación, la repetición y el abandono). La trayectoria escolar, por tanto, constituye un conjunto de factores y datos que afectan y muestran el comportamiento escolar de los estudiantes durante su estancia en la universidad (Cuevas, 2001). Dichos factores pueden ser de tipo psicológico y sociológico (cualitativos), o pueden proporcionar datos precisos sobre los resultados académicos de los estudiantes (cuantitativos).

Considerando las definiciones anteriores, se puede afirmar que la trayectoria académica se vincula con el recorrido de un grupo de estudiantes en un plan de estudios, los cuales generan información importante para la toma de decisiones de las instituciones educativas. 


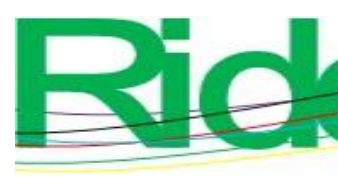

Revista Iberoamericana para la
Investigación y el Desarrollo Educativo
ISSN $2007-7467$

Rendimiento escolar

Para Galán (1985, citado por Romero, 2006) el rendimiento escolar es el grado de éxito o fracaso educativo fijado en función del logro de las metas académicas, en términos de objetivos de aprendizaje alcanzados y calificaciones individuales o grupales obtenidas. En otras palabras, el rendimiento académico se puede definir como el promedio de las calificaciones que los alumnos obtienen al finalizar el periodo escolar, y es un factor importante en el análisis de la calidad de una institución, ya que ofrece una aproximación a la realidad educativa.

Por ello, Chaín-Revuelta (1995) sugiere que los estudios de trayectorias escolares se convierten en una excelente plataforma para la instrumentación de mejoras en cuanto a objetivos, estrategias y cambios curriculares que permitan atender la deserción y el rezago, problemas de gran alcance internacional (Martínez-Rizo, 2001).

\section{Deserción escolar}

La deserción escolar se refiere a una situación en la que el estudiante se ausenta en repetidas ocasiones de las clases hasta que, finalmente, abandona el sistema educativo. Existen diversas causas por las que se da la deserción de los alumnos, entre las que se encuentran problemas económicos y familiares, situaciones académicas y sociales, etc.

\section{Metodología}

La presente investigación fue de tipo no experimental y se desarrolló por cohortes, es decir, conjunto de alumnos que ingresa a una carrera profesional en un mismo año (Cancela, Cea, Galindo y Valilla, 2016).

\section{Método y participantes}

El procedimiento que se llevó a cabo fue el análisis de la base de datos del Departamento de Control Escolar del Centro Universitario, de donde se consideraron los siguientes criterios de estudio, validados por dicha instancia: año en el que ingresaron y egresaron, asignaturas con mayor índice de reprobación (estos datos proporcionan el rezago de los alumnos), semestre en el que se imparten esas materias (para precisar dónde existe mayor complicación para los alumnos) y tipo de examen para acreditar cada asignatura.

En el estudio se analizaron 153 trayectorias académicas - proporcionadas por el Departamento de Control Escolar del Centro Universitario UAEM Valle de Méxicocorrespondientes a 76 estudiantes de ingeniería en Computación (ICO) y 77 alumnos de 

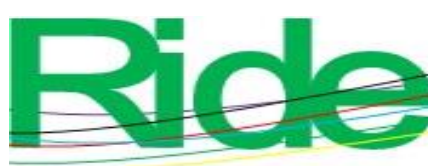

Revista Iberoamericana para la Investigación y el Desarrollo Educativo ISSN 2007 - 7467

ingeniería en Sistemas y Comunicaciones (ISC), los cuales pertenecen a la cohorte generacional 2014-2019. Del total, $26.4 \%$ son mujeres y $73.6 \%$ hombres. Sus rangos de edad oscilaban en los 17 y los 22 años.

\section{Resultados}

Con los datos proporcionados por el Departamento de Control Escolar del Centro Universitario UAEM Valle de México, se obtuvo que en ambas carreras 21 alumnos concluyeron su plan de estudios (27.6\% y $27.2 \%$, respectivamente): 15 hombres $(71.4 \%)$ y 6 mujeres (28.6 \%) en ingeniería en Computación (ICO), y 16 hombres (76.1\%) y 5 mujeres (23.8 \%) en ingeniería en Sistemas y Comunicaciones (ISC), (Figura 1).

Figura 1. Alumnos que concluyeron el plan de estudios

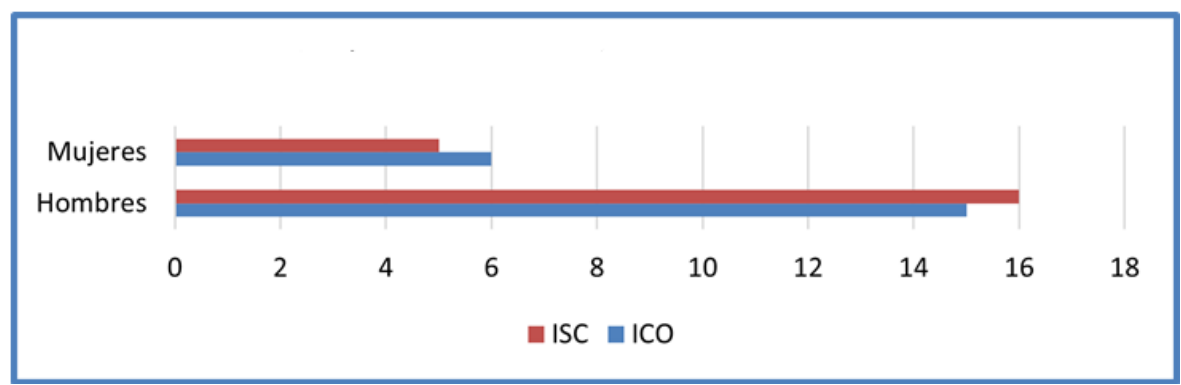

Fuente: Elaboración propia

\section{Grado de avance}

El grado de avance se define como la cantidad de alumnos que se encuentran inscritos en cada semestre. Ambas ingenierías comprenden 10 semestres (tabla 1).

Tabla 1. Porcentaje de grados de avance

\begin{tabular}{|c|c|c|c|c|c|c|c|c|c|c|}
\hline & $\mathbf{1 .}^{\circ}$ & $\mathbf{2 .}^{\circ}$ & $\mathbf{3 .}^{\circ}$ & $\mathbf{4 .}^{\circ}$ & $\mathbf{5 .}^{\circ}$ & $\mathbf{6 .}^{\circ}$ & $\mathbf{7 .}^{\circ}$ & $\mathbf{8 .}^{\circ}$ & $\mathbf{9 .}^{\circ}$ & $\mathbf{1 0 .}^{\circ}$ \\
\hline ICO & 96.2 & 83.6 & 89.3 & 91.7 & 92.3 & 95.4 & 95.3 & 97.2 & 97.5 & 98.3 \\
\hline ISC & 97.1 & 85.3 & 88.7 & 93.4 & 93.6 & 95.1 & 96.2 & 96.6 & 98.2 & 98.7 \\
\hline
\end{tabular}

Fuente: Elaboración propia

Como se puede observar en la tabla 1, el grado de avance por semestre de los alumnos se mantiene en un nivel alto para ambas carreras, aunque en segundo y tercero se percibe una disminución. Al respecto, se puede indicar que esos serían los momentos más difíciles de 


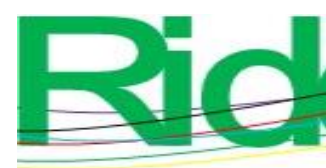

항

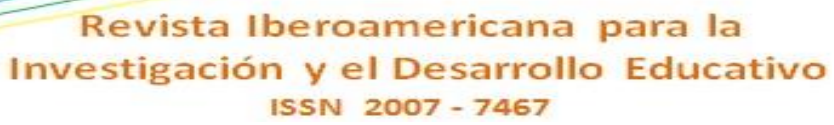

afrontar en las carreras debido a que se debe consolidar el proceso de adaptación. De hecho, al preguntar a los egresados sobre esta situación, la mayoría consideró el grado de complejidad de las materias, la falta de conocimientos o problemas personales.

\section{Deserción}

Otro indicador considerado en el estudio de las trayectorias de los alumnos fue la deserción, la cual se refiere al abandono de las materias o carrera en las cuales estaban inscritos los alumnos (figura 2).

Figura 2. Deserción

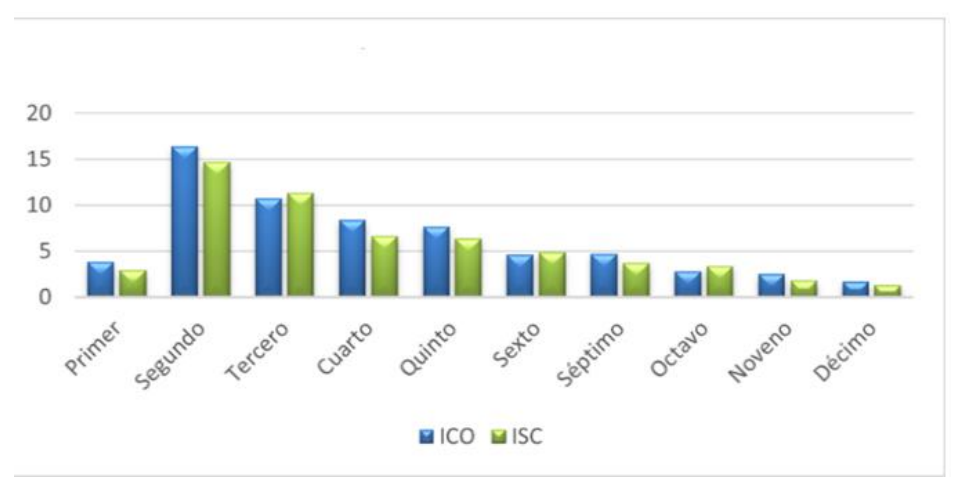

Fuente: Elaboración propia

En la figura 2 se observa que los niveles de deserción se presentan con mayor énfasis en el segundo y tercer semestre. Algunas de las causas más frecuentes para tomar esa decisión son la falta de identificación con la carrera, los problemas económicos, personales y académicos, y específicamente la cantidad de evaluaciones reprobadas durante su estancia en la institución (Vázquez Lozada, 2016).

\section{Reprobación}

La reprobación es el número o porcentaje de alumnos que no han obtenido los conocimientos necesarios en determinada asignatura, por lo que se ven en la necesidad de tener que recursarla (Instituto Nacional de Estadística y Geografía [Inegi], 2006). De acuerdo con los datos proporcionados por el Departamento de Control Escolar, las materias con mayor índice de reprobación en ingeniería en Computación (ICO) e ingeniería en Sistemas y Comunicaciones (ISC) se muestran en la tabla 2. 
Tabla 2. Materias con mayor índice de reprobación

\begin{tabular}{|c|c|c|}
\hline Área & Materia & Semestre \\
\hline \multicolumn{3}{|c|}{ Ingeniería en Sistemas y Comunicaciones } \\
\hline \multirow{6}{*}{ } & Álgebra y geometría analítica & Primero \\
\hline & Álgebra lineal & Segundo \\
\hline & Cálculo diferencial e integral & Segundo \\
\hline & Ecuaciones diferenciales & Tercero \\
\hline & Lógica matemática & Tercero \\
\hline & Cálculo vectorial & Cuarto \\
\hline Área & Materia & Semestre \\
\hline \multicolumn{3}{|c|}{ Ingeniería en Computación } \\
\hline \multirow{9}{*}{ } & Álgebra superior & Primero \\
\hline & Cálculo I & Primero \\
\hline & Geometría analítica & Primero \\
\hline & Física & Segundo \\
\hline & Álgebra lineal & Segundo \\
\hline & Cálculo II & Segundo \\
\hline & Cálculo III & Tercero \\
\hline & Ecuaciones diferenciales & Tercero \\
\hline & Análisis de Fourier & Cuarto \\
\hline
\end{tabular}

Fuente: Elaboración propia

Considerando lo anterior, las materias con mayor índice de reprobación son del área de ciencias básicas y matemáticas, ubicadas en los tres primeros semestres de ambas carreras, lo que se relaciona con la deserción detectada en esos semestres. Algunas de las causas de esta situación son el no contar con los conocimientos básicos, el grado de complejidad de la materia y por último el profesor.

Por otra parte, en la tabla 3 se muestra el tipo de examen (ordinario, extraordinario y título) mediante el cual los alumnos aprueban cuando están cursando por segunda vez la materia. Además, se enseña el porcentaje de alumnos que aprueban en cada tipo de examen y el rango de calificaciones que obtienen, el cual es bajo, a pesar de cursarla por segunda ocasión. 


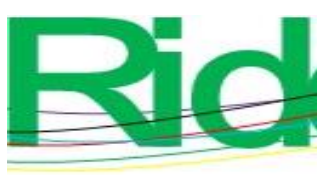

Revista Iberoamericana para la Investigación y el Desarrollo Educativo ISSN 2007 - 7467

Tabla 3. Tipo de examen mediante el cual los alumnos aprueban en un segundo curso

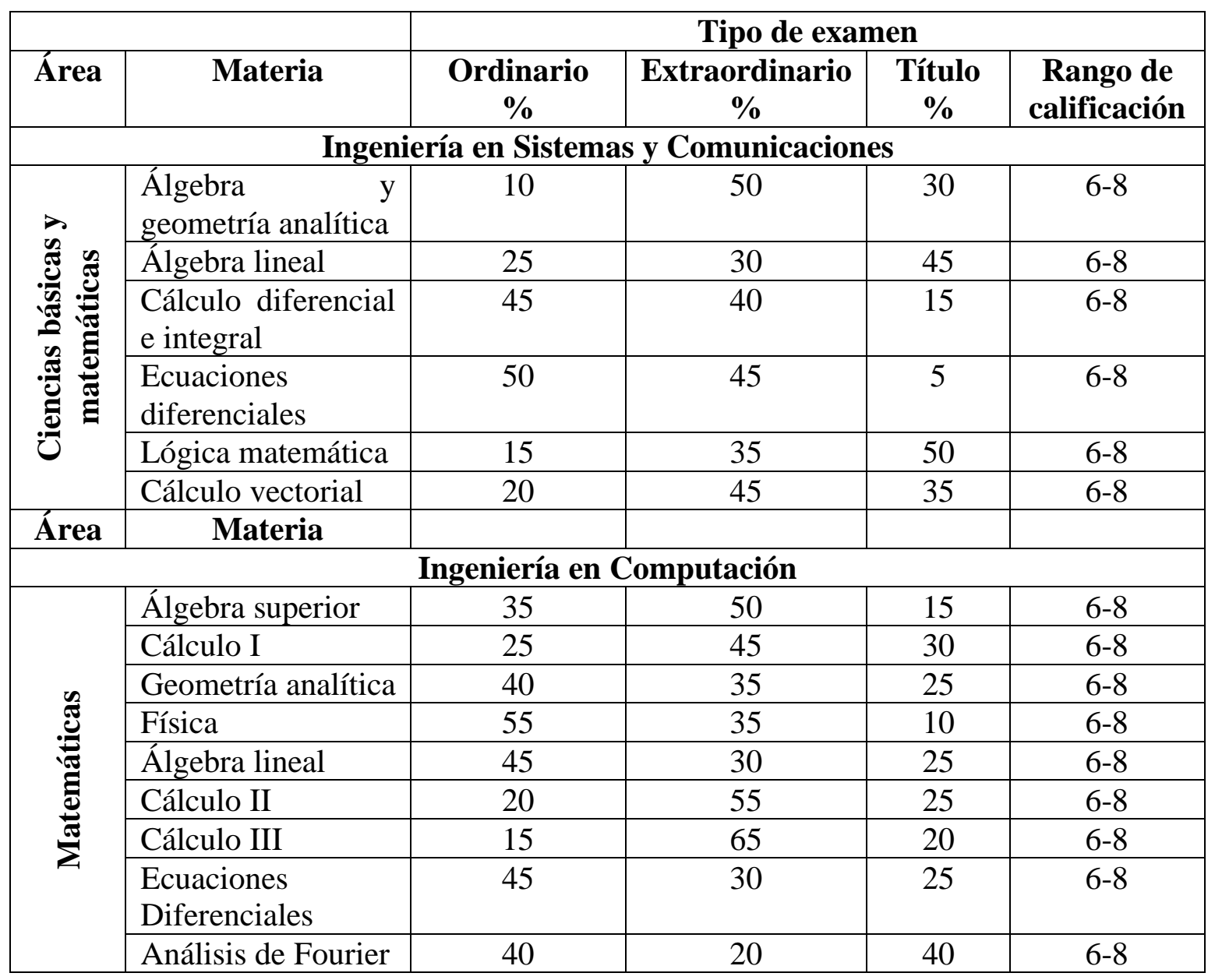

Fuente: Elaboración propia

Finalmente, la información proporcionada por el Departamento de Control Escolar también permitió conocer la escuela de donde procedían los alumnos de ambas ingenierías (tabla 4), dato que podría resultar significativo, ya que se podría establecer algún contacto con las autoridades de esas instituciones para mostrarles las áreas de oportunidad en el área de matemáticas. 


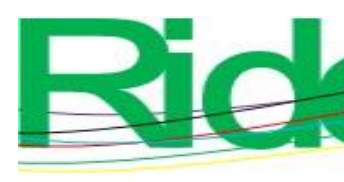

Revista Iberoamericana para la Investigación y el Desarrollo Educativo ISSN 2007 - 7467

Tabla 4. Escuelas de procedencia de los alumnos de ICO e ISC

\begin{tabular}{|c|c|}
\hline $\begin{array}{c}\text { Ingeniería en Sistemas y } \\
\text { Comunicaciones }\end{array}$ & $\begin{array}{l}\text { - Centro de Estudios Tecnológicos } \\
\text { Industrial y de Servicios nro. } 35 \\
\text { - Colegio de Estudios Científicos y } \\
\text { Tecnológicos del Estado de México, } \\
\text { plantel Nicolás Romero } \\
\text { - Escuela Preparatoria Oficial nro. } 148 \\
\text { - Escuela Preparatoria Oficial nro. } 87 \\
\text { - Colegio de Bachilleres nro. } 5\end{array}$ \\
\hline Ingeniería en Computación & $\begin{array}{l}\text { - Colegio de Bachilleres nro. } 5 \\
\text { - Colegio de estudios Científicos y } \\
\text { Tecnológicos del Estado de México, } \\
\text { plantel Nicolás Romero } \\
\text { - Escuela Preparatoria Oficial nro. } 87 \\
\text { - CBTS Atizapán de Zaragoza } \\
\text { - Centro de Estudios Tecnológicos } \\
\text { Industrial y de Servicios nro. } 35 \\
\text { - Nueva Escuela Tecnológica Nicolás } \\
\text { Romero. }\end{array}$ \\
\hline
\end{tabular}

Fuente: Elaboración propia

\section{Discusión}

Los resultados muestran que la demanda de ambas ingenierías es equivalente, aunque en cuanto al sexo existe una preponderancia por parte de los hombres. Asimismo, se puede indicar que el grado de avance disminuye en el segundo y tercer semestre de ambas carreras, ya que los alumnos consideran que son los de mayor grado de dificultad. Esta situación se genera porque los estudiantes carecen de bases sólidas para encarar las materias pertenecientes a las ciencias básicas, lo que provoca que aumenten los niveles de reprobación y rezago, pues algunas de esas asignaturas tienen seriación. De hecho, Programación es una de las materias que más se les dificulta, la cual tiene una seriación mayor.

Por otra parte, el no identificarse con la carrera, así como los problemas económicos, personales y académicos son las causas del abandono de los estudios. Asimismo, se podría considerar como dato importante la escuela de procedencia de los alumnos de ambas ingenierías para comunicar a sus respectivas autoridades las áreas de oportunidad en las que pueden apoyar a sus estudiantes. 


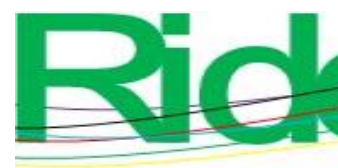
Revista Iberoamericana para la
Investigación y el Desarrollo Educativo
ISSN $2007-7467$

\section{Conclusiones}

El estudio y seguimiento de las trayectorias académicas en las instituciones de educación superior constituye una fuente de información esencial para mejorar la calidad educativa. Esto se debe a que al analizar las trayectorias se pueden identificar los niveles de riesgo en los que se encuentran los alumnos, lo cual podría servir para crear estrategias de apoyo. En tal sentido, las soluciones que se proponen se explican a continuación:

\section{Mentoría académica}

Esta estrategia consiste es ofrecer asesoría disciplinaria a través de alumnos sobresalientes, específicamente en temas que representen riesgo académico, así como recomendación de estrategias de aprendizaje y de estudio para mejorar el aprovechamiento escolar mediante una interacción alumno-alumno.

\section{Asesorías}

Las asesorías pueden ser ofrecidas por un profesor diferente al que imparte la materia. Estas procurarían apoyar a los alumnos en una situación problemática y resolver dudas académicas. Los estudiantes solicitarían asesoría a través de su tutor, directamente con el coordinador de tutoría o con el profesor.

\section{Tutorías}

Las tutorías serían estrategias de atención personalizada para disminuir los índices de deserción y contribuir a la formación integral del alumno. En este sentido, se propone que los tutores cumplan con sus diferentes actividades administrativas y académicas para apoyar a los alumnos en su transitar por el programa educativo.

\section{Canalización psicológica}

En algunas ocasiones el bajo desempeño académico de los alumnos se debe no solo a la incomprensión de los temas de las materias, sino también a problemas personales, por lo que requieren atención psicológica. Por ello, el tutor debe poner en contacto al alumno con la instancia pertinente. 


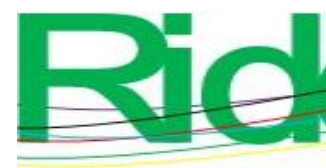
Revista Iberoamericana para la
Investigación y el Desarrollo Educativo
ISSN $2007-7467$

\section{Comprensión lectora}

La comprensión de textos es esencial para el aprendizaje debido a que la mayoría de la información recibida proviene de fuentes escritas. Por eso, la aplicación de estrategias didácticas en esta área contribuiría a disminuir los índices de reprobación.

\section{Actualización docente}

La actualización docente es un factor importante para desarrollar actitudes y aptitudes útiles para potenciar las capacidades de los estudiantes en cuanto a la solución de problemas.

En síntesis, se puede concluir que los indicadores de trayectorias escolares ofrecen mecanismos que sirven para proponer estrategias que se podrían implementar para atender a los estudiantes durante todo su recorrido académico.

\section{Trabajos futuros}

Algunas líneas de investigación que se podrían desarrollar en trabajos futuros son las siguientes:

- Actualizar el Sistema de Información de Tutoría Académica para contar con una herramienta de gestión de información académica que sistematice, dé seguimiento y evalúe la actividad tutorial. Esto sería útil para que el alumno tuviera contacto con su tutor, mientras que a este último le serviría para apoyar de manera oportuna al alumno a través del análisis de su historial académico.

- Integrar en el Sistema de Control Escolar un apartado que permita a los coordinadores de carrera obtener información de la trayectoria de los alumnos de una manera sencilla y gráfica. De este modo se podrían identificar las materias cursadas, los segundos cursos, las materias reprobadas, las pendientes, el grado de avanece, el estatus de los alumnos, etc. 


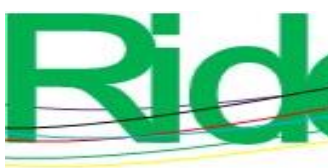
Revista Iberoamericana para la
Investigación y el Desarrollo Educativo
ISSN $2007-7467$

\section{Referencias}

Bartolucci, J. (1994). Desigualdad social, educación superior y sociología en México. México: Porrúa.

Bartolucci, J. (1998). Posición social, trayectoria escolar y elección de carrera (seguimiento de una generación de estudiantes de la UNAM, 1976-1985). En Marsiske, R. (coord.), Los estudiantes. Trabajos de historia y sociología (pp. 291-360). México: Centro de Estudios Sobre la Universidad-Universidad Nacional Autónoma de México/Plaza y Valdés.

Calatayud, A. y Merino, C. (1984). Los perfiles escolares en la UNAM. Revista Perfiles Educativos, 25(6), 16-29.

Camarena, R. et al. (1985), "Reflexiones en torno al rendimiento escolar y a la eficiencia terminal", en Revista de la Educación Superior, $N^{\circ}$ 53, ANUIES, México., Recuperado de http://publicaciones.anuies.mx/revista/53/1/2/es/reflexiones-entorno-al-rendimiento-escolar

Cancela, G., Cea, M., Galindo, L., \& Valilla, G. (2016). www.uam.es. Obtenido de www.uam.es:https://www.uam.es/personal_pdi/stmaria/jmurillo/InvestigacionEE/Pr esentaciones/Curso_10/ EX-POST-FACTO_Trabajo.pdf

Chaín-Revuelta, R. (1995). Estudiantes universitarios. Trayectorias escolares. México: Universidad Veracruzana y Universidad Autónoma de Aguascalientes.

Chaín-Revuelta, R. (2001). Demanda, estudiantes y elección. México: Universidad Veracruzana.

Chaín-Revuelta, R. y Ramírez, C. (1997). Trayectoria escolar: un estudio sobre la eficiencia terminal en la Universidad Veracruzana. Revista de la Educación Superior, 15(102), 79-98.

Chaín-Revuelta, R., Cruz Ramírez, N., Martínez Morales, M. y Jácome Ávila, N. (2003). Examen de selección y probabilidad de éxito escolar en estudios superiores. Estudio en una universidad pública estatal mexicana. REDIE. Revista Electrónica de Investigación Educativa. Recuperado de http://www.redalyc.org/articulo.oa?id=15505105

Covo, M. (1988). Reflexiones sobre el estudio de la deserción en México. México: ANUIESSEP. 


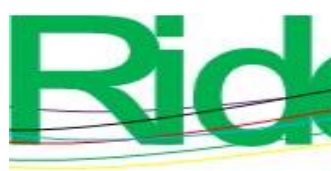

Revista Iberoamericana para la Investigación y el Desarrollo Educativo ISSN $2007-7467$

Cuevas, M. (2001). La trayectoria escolar universitaria: un acercamiento desde las habilidades básicas de pensamiento y el rendimiento académico. Revista DIDAC, (38), 45-49.

De Allende, C. y Gómez V. (1989). Bibliografía comentada sobre la trayectoria escolar. En Asociación Nacional de Universidades e Instituciones de Educación Superior (Anuies), Trayectoria escolar en la educación superior. Panorámica de la investigación y acercamientos metodológicos. México: SEP-ANUIES.

Fernández A., Peña A. y Vera F. (2006). Los estudios de trayectoria escolar. Su aplicación en la educación media superior. Revista de la Facultad de Filosofía y Letras, (6), 2429. Recuperado de http://dialnet.unirioja.es/servlet/articulo?codigo=2290466

González, M. (1999). Seguimiento de trayectorias escolares. México: ANUIES.

Instituto Nacional de Estadística y Geografía [Inegi] (2006). Encuesta Nacional de Ocupación $\quad y \quad$ Empleo. Recuperado de http://www.inegi.org.mx/sistemas/biblioteca/detalle

Gómez, S. (1990). Estudio exploratorio en estudiantes universitarios. Referencias sociodemográficas y elección de carrera. Práxis educativa.

Martínez, R. (2001). Sistemas de información para el estudio de trayectorias escolares. En ANUIES, Deserción, rezago y eficiencia terminal en la IES Propuesta metodológica para su estudio (págs. 75-93). México: ANUIES..

Moreno, Z. M., Medina, C. L., Espinoza, A. D. y Miranda, G. D. (2005). Bases para el Modelo de Innovación Curricular. Toluca: UAEM.

Noriega, P.(1989). El rendimiento escolar en ciencias básicas y su mejoramiento a través de condiciones de estudio apropiadas para el alumno. Revista de la Educación Superior. ANUIES. Vol. XVIII (2). No.70. México, D.F.

Ponce de León, M. (2003). Guía para el seguimiento de trayectorias escolares. Universidad Autónoma del Estado de Hidalgo. Recuperado de http://intranet.uaeh.edu.mx/DGP/pdf/2_guia_trayectoria.pdf

Reyes Pérez, V. (2006). Informe de evaluación del cuestionario para alumnos de primer ingreso a licenciatura (tesis de maestría). México, UNAM-Facultad de Psicología.

Romero, B. (2006). Autoconcepto y rendimiento escolar en adolescentes que cursan el tercer grado de la educación media básica (tesis de licenciatura). Toluca: Universidad Autónoma del Estado de México.

Rugarcía, A. (1994). La calidad del posgrado en México. Renglones, (29), 57-62. 


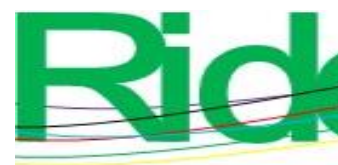

Revista Iberoamericana para la
Investigación y el Desarrollo Educativo
ISSN $2007-7467$

Sánchez, D. (2006), El proceso de graduación en el posgrado de Pedagogía de la UNAM: el caso de la maestría en Pedagogía, Tesis Doctoral, México, UNAM-Facultad de Filosofía y Letras.

Salmi, J. (2016). Combatir la deserción en la educación superior: lecciones a partir de la experiencia internacional. Recuperado de http://noticias.universia.net.mx/ educacion/noticia/2016/11/24/1146666/pautas-combatir-desercion-educacionsuperior.html

Tinto, V. (1993). Leaving College; Rethinking the Causes and Cures of Student Attrition, (2 ${ }^{\text {th }}$ ed.). Chicago: University of Chicago Press.

Universidad Autónoma de Tamaulipas (UAT) (2016). 3. ${ }^{\text {er }}$ informe rectoral 2016. Tamaulipas, UAT. Recuperado de https:// www.uat.edu.mx/TRANS/VIIndicadoresderesultados/ Informe\%20Rectoral\%202016.pdf

Universidad Autónoma del Estado de México [UAEM]. (2009). Plan General de Desarrollo 2009-2021. Toluca: Universidad Autónoma del Estado de México.

Universidad Autónoma del Estado de México [UAEM]. (2017a). Plan Rector de Desarrollo Institucional 2009-2013. Toluca: Universidad Autónoma del Estado de México.

Universidad Autónoma del Estado de México [UAEM]. (2017b). Plan de Desarrollo del Centro Universitario Valle de México 2017-2021. Atizapán de Zaragoza: Universidad Autónoma del Estado de México.

Universidad Nacional Autónoma de México (UNAM) (2015). Historias académicas de alumnos. México, Subdirección Técnica, Coordinación de la Administración Escolar (CAE)-UNAM.

Vázquez Lozada, S. (2016). Principales causas de deserción de la Licenciatura en Geografía. UAEM. 


\begin{tabular}{|c|c|}
\hline Rol de Contribución & Autor (es) \\
\hline Conceptualización & Ivonne Rodríguez Pérez \\
\hline Metodología & $\begin{array}{l}\text { Ivonne Rodríguez Pérez (Igual), José Martín Flores } \\
\text { Albino(Igual). }\end{array}$ \\
\hline Software & NO APLICA \\
\hline Validación & $\begin{array}{l}\text { Rigoberto Pérez Ramírez (Igual), José Martín Flores Albino } \\
\text { (Igual) }\end{array}$ \\
\hline Análisis Formal & $\begin{array}{l}\text { Ivonne Rodríguez Pérez (Igual), Rigoberto Pérez Ramírez } \\
\text { (Igual), José Martín Flores Albino (Igual) }\end{array}$ \\
\hline Investigación & $\begin{array}{l}\text { Ivonne Rodríguez Pérez (Principal), Rigoberto Pérez Ramírez } \\
\text { (Apoyo) }\end{array}$ \\
\hline Recursos & Departamento de Control Escolar del Espacio Académico \\
\hline Curación de datos & José Martín Flores Albino \\
\hline $\begin{array}{l}\text { Escritura - Preparación del } \\
\text { borrador original }\end{array}$ & $\begin{array}{l}\text { Ivonne Rodríguez Pérez (Igual), Rigoberto Pérez Ramírez } \\
\text { (Igual), José Martín Flores Albino (Igual) }\end{array}$ \\
\hline $\begin{array}{l}\text { Escritura - Revisión y } \\
\text { edición }\end{array}$ & $\begin{array}{l}\text { Ivonne Rodríguez Pérez (Igual), Rigoberto Pérez Ramírez } \\
\text { (Igual), José Martín Flores Albino (Apoyo) }\end{array}$ \\
\hline Visualización & $\begin{array}{l}\text { Ivonne Rodríguez Pérez (Principal), Rigoberto Pérez Ramírez } \\
\text { (Igual), José Martín Flores Albino (Igual) }\end{array}$ \\
\hline Supervisión & $\begin{array}{l}\text { Ivonne Rodríguez Pérez (Igual, Rigoberto Pérez Ramírez } \\
\text { (Igual), José Martín Flores Albino (Igual) }\end{array}$ \\
\hline $\begin{array}{l}\text { Administración de } \\
\text { Proyectos }\end{array}$ & $\begin{array}{l}\text { Ivonne Rodríguez Pérez (Principal), Rigoberto Pérez Ramírez } \\
\text { (Igual), José Martín Flores Albino (Apoyo) }\end{array}$ \\
\hline Adquisición de fondos & $\begin{array}{l}\text { Ivonne Rodríguez Pérez (Igual), Rigoberto Pérez Ramírez } \\
\text { (Igual), José Martín Flores Albino (Igual) }\end{array}$ \\
\hline
\end{tabular}

